\title{
Note About Non-Relativistic Diffeomorphism Invariant Gravity Action in Three Dimensions
}

\author{
Josef Klusoñ 1 \\ Department of Theoretical Physics and Astrophysics \\ Faculty of Science, Masaryk University \\ Kotlárská 2, 611 37, Brno \\ Czech Republic
}

\begin{abstract}
This short note is devoted to Hamiltonian analysis of three dimensional gravity action that was proposed recently in arXiv:1309.7231. We perform this analysis and determine the number of physical degrees of freedom.
\end{abstract}

\footnotetext{
${ }^{1}$ E-mail: klu@physics.muni.cz
} 


\section{Introduction}

Recently new form of three dimensional gravity action was proposed in [1]. This action was derived from relativistic three dimensional gravity action by new limiting procedure which however shares some similarity with the analysis performed in [2] and also in [8]2. Among other new results it was further argued in [1] that given action is invariant under non-relativistic diffeomorphism whose explicit form is given in (2).

The form of this three dimensional gravity action is very interesting and certainly deserves further investigation. In particular, it would be interesting to find the Hamiltonian formulation of given action. To do this we rewrite the action found in [1] to more familiar form which is similar to the three dimensional non-relativistic action studied in [8]. However there is an important difference between these actions since the action studied in [8] is invariant under foliation preserving diffeomorphism which is more general than (2) since in addition to the rules given in (2) it contains the transformation $t^{\prime}=f(t)$. As a result the Hamiltonian formulation of the action analyzed in [8] possesses the global Hamiltonian constraint which, as we will see, is absent in case of the action given in [1]. On the other hand the presence of the global constraint does not restrict the number of the local degrees of freedom. Explicitly, when we perform the Hamiltonian analysis of the action given in [1] and identify all constraints we find that there are no local degrees of freedom with agreement with the number of physical degrees of freedom of three dimensional non-relativistic covariant Hořava-Lifshitz gravity [8].

\section{New Non-Relativistic Gravity Action}

In [1] new form of non-relativistic $2+1$ gravity action was found in the form

$$
S=m \int d t d^{2} \mathbf{x} \sqrt{g}\left[-\frac{1}{4} \dot{g}^{i j} \dot{g}_{i j}-\frac{1}{4}\left(g^{i j} \dot{g}_{i j}\right)^{2}-\frac{1}{m}\left(A^{i} \partial_{i} \ln g-\dot{g}^{i j} \nabla_{i} A_{j}+A_{0} R\right)+\frac{1}{4 m^{2}} F_{i j} F^{i j}\right]
$$

where $F_{i j}=\partial_{i} A_{j}-\partial_{j} A_{i}$. Note that this theory lives on a 2-dimensional surface without boundaries with the metric tensor $g_{i j}$, where $g=\operatorname{det} g_{i j}, R$ is corresponding scalar curvature and where $\nabla_{i}$ is covariant derivative compatible with the metric $g_{i j}$.

Before we proceed to the Hamiltonian formalism we would like to explicitly check whether this action is invariant under non-relativistic diffeomorphism

$$
\begin{aligned}
A_{0}^{\prime}\left(\mathbf{x}^{\prime}, t\right) & =A_{0}(\mathbf{x}, t)-A_{i}(\mathbf{x}, t) \partial_{t} \xi^{i}(\mathbf{x}, t) \\
A_{i}^{\prime}\left(\mathbf{x}^{\prime}, t\right) & =A_{i}(\mathbf{x}, t)-A_{j}(\mathbf{x}, t) \partial_{i} \xi^{j}(\mathbf{x}, t)-m g_{i j}(\mathbf{x}, t) \partial_{t} \xi^{j}(\mathbf{x}, t) \\
g_{i j}^{\prime}\left(\mathbf{x}^{\prime}, t\right) & =g_{i j}(\mathbf{x}, t)-\partial_{i} \xi^{k} g_{k j}(\mathbf{x}, t)-g_{i k} \partial_{j} \xi^{k}(\mathbf{x}, t)
\end{aligned}
$$

\footnotetext{
${ }^{2}$ For review of Hořava-Lifshitz gravity, see [3, 4, 5].
} 
where

$$
\delta t \equiv t^{\prime}-t=0, \quad \delta x^{i} \equiv x^{i}-x^{i}=\xi^{i}(t, \mathbf{x}) .
$$

For future purposes we rewrite the action (1) into more familiar form. First of all we replace $\dot{g}^{i j}$ with

$$
\dot{g}^{i j}=-g^{i m} \dot{g}_{m n} g^{n j}
$$

using the definition of the metric $g^{i j}$ as inverse to $g_{i j}$. Then the kinetic term takes the form

$$
g^{i k} g^{i l} \dot{g}_{i j} \dot{g}_{k l}-g^{i j} g^{k l} \dot{g}_{i j} \dot{g}_{k l}=\dot{g}_{i j} \mathcal{G}^{i j k l} \dot{g}_{k l},
$$

where

$$
\mathcal{G}^{i j k l}=\frac{1}{2}\left(g^{i k} g^{j l}+g^{i l} g^{j k}\right)-g^{i j} g^{k l},
$$

with inverse

$$
\mathcal{G}_{i j k l}=\frac{1}{2}\left(g_{i k} g_{j l}+g_{i l} g_{j k}\right)-g_{i j} g_{k l}, \quad \mathcal{G}_{i j k l} \mathcal{G}^{k l m n}=\frac{1}{2}\left(\delta_{i}^{k} \delta_{j}^{l}+\delta_{i}^{l} \delta_{j}^{k}\right) .
$$

Further, using integration by parts we observe that we can perform following replacement in the action (1)

$$
-\int d^{2} \mathbf{x} \sqrt{g} A^{i} \partial_{t} \partial_{i} \ln g=\int d^{2} \mathbf{x} \partial_{i}\left[\sqrt{g} A^{i}\right] \partial_{t} \ln g=\int d^{2} \mathbf{x} \partial_{i}\left[\sqrt{g} A^{i}\right] \dot{g}_{k l} g^{k l} .
$$

Observe that $\sqrt{g} A^{i}$ is tensor density of weight 1 so that

$$
\nabla_{i}\left(\sqrt{g} A^{i}\right)=\partial_{i}\left(\sqrt{g} A^{i}\right) .
$$

Now with the help of these results we find that the action (11) can be rewritten into the form

$$
\begin{aligned}
S & =m \int d t d^{2} \mathbf{x} \sqrt{g}\left[\tilde{K}_{i j} \mathcal{G}^{i j k l} \tilde{K}_{k l}-\frac{1}{m} A_{0} R-\right. \\
& \left.-\frac{1}{4 m^{2}}\left(\nabla_{i} A_{j}+\nabla_{j} A_{i}\right) \mathcal{G}^{i j k l}\left(\nabla_{k} A_{l}+\nabla_{l} A_{k}\right)+\frac{1}{4 m^{2}} F_{i j} F^{i j}\right],
\end{aligned}
$$

where we defined $\tilde{K}_{i j}$ as

$$
\tilde{K}_{i j}=\frac{1}{2}\left(\partial_{t} g_{i j}-\frac{1}{m} \nabla_{i} A_{j}-\frac{1}{m} \nabla_{j} A_{i}\right) .
$$

Finally we use the fact that

$$
\int d^{2} \mathbf{x} \sqrt{g} \nabla_{i} A_{j} g^{i j} \nabla_{k} A_{l} g^{k l}=\int d^{2} \mathbf{x} \sqrt{g}\left(\nabla_{k} A_{j} g^{j i} \nabla_{i} A_{l} g^{l k}+\frac{A_{i} A^{i}}{2} R\right),
$$


where we performed integration by parts and used the definition

$$
\nabla_{i} \nabla_{j} A_{k}-\nabla_{j} \nabla_{i} A_{k}=R_{i j k}{ }^{l} A_{l}
$$

together with the fact that two dimensional Riemann tensor has the form

$$
R_{i j k l}=\frac{R}{2}\left(g_{i k} g_{j l}-g_{i l} g_{k j}\right),
$$

where $R$ is the scalar curvature. As a result we find that the action (10) has the form

$$
S=m \int d t d^{2} \mathbf{x} \sqrt{g}\left[\tilde{K}_{i j} \mathcal{G}^{i j k l} \tilde{K}_{k l}-\frac{1}{m}\left(A_{0}-\frac{A_{i} A^{i}}{2 m}\right) R\right] .
$$

Now we explicitly show that this action is invariant under (2). To begin with note that $\tilde{K}_{i j}$ transform under (2) as

$$
K_{i j}^{\prime}\left(\mathbf{x}^{\prime}, t\right)=K_{i j}(\mathbf{x}, t)-\partial_{i} \xi^{k} K_{k j}(\mathbf{x}, t)-K_{i k} \partial_{j} \xi^{k}(\mathbf{x}, t)
$$

and hence

$$
\left(K_{i j} \mathcal{G}^{i j k l} K_{k l}\right)^{\prime}\left(\mathbf{x}^{\prime}, t\right)=K_{i j}(\mathbf{x}, t) \mathcal{G}^{i j k l}(\mathbf{x}, t) K_{k l}(\mathbf{x}, t) .
$$

Further, using (21) we find

$$
\left(A_{0}-\frac{1}{2 m} A_{i} A^{i}\right) R\left(\mathbf{x}^{\prime}, t^{\prime}\right)=\left(A_{0}-\frac{1}{2 m} A_{i} A^{i}\right) R(\mathbf{x}, t)
$$

Then it is easy to see that the action (15) is invariant under (2).

We can also derive the action (15) directly when we implement the limiting procedure suggested in [1] to the case of three dimensional relativistic gravity action written in $2+1$ formalism. Explicitly we start with the action

$$
S=m c^{2} \int d t d^{2} \mathbf{x} \sqrt{-g^{(3)}}(3) R,
$$

where $m$ is mass scale and $c$ is speed of light, $g^{(3)}$ is three dimensional metric and ${ }^{(3)} R$ is corresponding curvature. Following [1] we choose the parameterization of the metric $g_{\mu \nu}^{(3)}$ as

$$
g_{\mu \nu}^{(3)}=\left(\begin{array}{cc}
-1+\frac{2 A_{0}}{m c^{2}} & \frac{A_{i}}{m c} \\
\frac{A_{i}}{m c} & g_{i j}
\end{array}\right) .
$$

Now we compare (20) with the $2+1$ decomposition of the metric $g_{\mu \nu}^{(3)}[\underline{6}, 7]$

$$
\begin{aligned}
& { }^{(3)} g_{00}=-N^{2}+N_{i} g^{i j} N_{j}, \quad{ }^{(3)} g_{0 i}=N_{i}, \quad{ }^{(3)} g_{i j}=g_{i j}, \\
& { }^{(3)} g^{00}=-\frac{1}{N^{2}}, \quad{ }^{(3)} g^{0 i}=\frac{N^{i}}{N^{2}}, \quad{ }^{(3)} g^{i j}=g^{i j}-\frac{N^{i} N^{j}}{N^{2}} .
\end{aligned}
$$


Further, three dimensional scalar curvature in $2+1$ formalism has the form

$$
{ }^{(3)} R=K_{i j} \mathcal{G}^{i j k l} K_{k l}+R,
$$

where

$$
K_{i j}=\frac{1}{2 N}\left(\frac{\partial g_{i j}}{\partial x^{0}}-\nabla_{i} N_{j}-\nabla_{j} N_{i}\right),
$$

and where $\nabla_{i}$ are covariant derivatives defined by the metric $g_{i j}$ and where we ignored the boundary terms. When we identify (20) and (21) we find

$$
N_{i}=\frac{A_{i}}{m c}, \quad N^{2}=1-\frac{2 A_{0}}{m c^{2}}+\frac{A_{i} g^{i j} A_{j}}{m^{2} c^{2}} .
$$

Now we are ready to proceed with the limiting procedure when we start with the action in $2+1$ formalism

$$
S=m c^{2} \int d^{3} \mathbf{x} \sqrt{g} N\left[K_{i j} \mathcal{G}^{i j k l} K_{k l}+R\right] .
$$

Using (24) we find that the action (25) in the limit $c \rightarrow \infty$ takes the form

$$
\begin{aligned}
S & =m \int d t d^{2} \mathbf{x} \sqrt{g}\left[\frac{1}{4}\left(\dot{g}_{i j}-\frac{1}{m} \nabla_{i} A_{j}-\frac{1}{m} \nabla_{j} A_{i}\right) \mathcal{G}^{i j k l}\left(\dot{g}_{k l}-\frac{1}{m} \nabla_{k} A_{l}-\frac{1}{m} \nabla_{l} A_{k}\right)-\right. \\
& \left.-\frac{1}{m}\left(\frac{A_{0}}{m}-\frac{A_{i} g^{i j} A_{j}}{2 m}\right) R\right]
\end{aligned}
$$

with complete agreement with the action (15).

Now we can proceed to the Hamiltonian analysis of (26). In fact, this analysis is rather straightforward and closely resembles the analysis performed in case of non-relativistic covariant Hořava-Lifshitz gravity [8, 9, 10]. Explicitly, from (26) we find following momenta

$$
\pi^{i j}=\frac{\delta L}{\delta \partial_{t} g_{i j}}=2 m \sqrt{g} \mathcal{G}^{i j k l} \tilde{K}_{k l}, \quad \pi^{i}=\frac{\delta L}{\delta \partial_{t} A_{i}} \approx 0, \quad \pi^{0}=\frac{\delta L}{\delta \partial_{t} A_{0}} \approx 0 .
$$

Then it is easy to see that the Hamiltonian has the form

$$
H=\int d^{2} \mathbf{x}\left(\mathcal{H}_{0}+\frac{1}{m} A_{i} g^{i j} \mathcal{H}_{j}+v_{0} \pi^{0}+v_{i} \pi^{i}\right),
$$

where

$$
\begin{aligned}
& \mathcal{H}_{0}=\frac{1}{m \sqrt{g}} \pi^{i j} \mathcal{G}_{i j k l} \pi^{k l}+\sqrt{g}\left(\frac{A_{0}}{m}-\frac{A_{i} g^{i j} A_{j}}{2 m}\right) R, \\
& \mathcal{H}_{i}=-2 g_{i k} \nabla_{j} \pi^{k j},
\end{aligned}
$$

and where the primary constraints $\pi^{i} \approx 0, \pi^{0} \approx 0$ were included. 
Now the requirement of the preservation of the primary constraints $\pi^{0} \approx 0, \pi^{i} \approx$ 0 implies following secondary constraints

$$
\begin{aligned}
& \partial_{t} \pi^{0}=\left\{\pi^{0}, H\right\}=-\frac{\sqrt{g}}{m} R \equiv-\frac{1}{m} \mathcal{G}^{0} \approx 0, \\
& \partial_{t} \pi^{i}=\left\{\pi^{i}, H\right\}=-\frac{1}{m} g^{i j} \mathcal{H}_{j}+\frac{\sqrt{g}}{m} g^{i j} A_{j} R \approx-\frac{1}{m} g^{i j} \mathcal{H}_{j} \approx 0 .
\end{aligned}
$$

Then the total Hamiltonian with all constraints included has the form

$$
H_{T}=\int d^{2} \mathbf{x}\left(\mathcal{H}_{0}+v_{0} \pi^{0}+v_{i} \pi^{i}+\Gamma_{0} \mathcal{G}^{0}+\Gamma^{i} \mathcal{H}_{i}\right)
$$

where $v_{0}, v_{i}, \Gamma_{0}, \Gamma^{i}$ are Lagrange multipliers corresponding to the constraints $\pi^{0}, \pi^{i}, \mathcal{G}^{0}, \mathcal{H}_{i}$.

As the final step we check the stability of all constraints. First of all the constraints $\pi^{0}, \pi^{i}$ are trivially preserved that implies that they are the first class constraints. Further we introduce the smeared form of the constraint $\mathcal{H}_{i}$

$$
\mathbf{T}_{S}\left(N^{i}\right)=\int d^{2} \mathbf{x} N^{i} \mathcal{H}_{i}
$$

Then it is easy to show that $\mathbf{T}_{S}\left(N^{i}\right)$ is generator of the spatial diffeomorphism with following Poisson brackets

$$
\begin{aligned}
\left\{\mathbf{T}_{S}\left(N^{i}\right), \mathbf{T}_{S}\left(M^{j}\right)\right\} & =\mathbf{T}_{S}\left(N^{j} \partial_{j} M^{i}-M^{j} \partial_{j} N^{i}\right) \\
\left\{\mathbf{T}_{S}\left(N^{i}\right), \mathcal{G}^{0}\right\} & =-N^{i} \partial_{i} \mathcal{G}^{0}-\partial_{i} N^{i} \mathcal{G}^{0}
\end{aligned}
$$

This result also implies that the requirement of the preservation of the constraints $\mathcal{H}_{i}$ during the time evolution of the system does not generate any additional constraint. Finally the time development of the constraint $\mathcal{G}^{0}$ is given following equation

$$
\partial_{t} \mathcal{G}^{0}=\left\{\mathcal{G}^{0}, H_{T}\right\}=-\frac{1}{m} \nabla_{m} g^{m n} \mathcal{H}_{n} \approx 0
$$

using

$$
\delta R=\nabla^{i} \nabla^{j} \delta g_{i j}-g^{i j} \nabla^{k} \nabla_{k} \delta g_{i j}-R^{i j} \delta g_{i j}, \quad R_{i j}=\frac{R}{2} g_{i j} .
$$

Equation (34) tells us that $\mathcal{G}^{0}$ is preserved during the time evolution. Further, since it weakly commutes with all other constraints it is the first class constraint.

Let us conclude our results. We have following collections of the first class constraints $\pi^{i} \approx 0, \pi^{0} \approx 0, \mathcal{H}_{i} \approx 0$ and $\mathcal{G}^{0} \approx 0$. The constraints $\pi^{i} \approx 0, \pi^{0} \approx 0$ can be gauge fixed so that $A_{i}, A_{0}$ and their conjugate momenta are eliminated. At the same way by gauge fixing $\mathcal{H}_{i}, \mathcal{G}^{0}$ we eliminate all degrees of freedom corresponding 
to three dimensional metric $g_{i j}$. In other words there are no physical degrees of freedom left. This result is in agreement with the number of physical degrees of freedom in three dimensional non-relativistic covariant Hořava-Lifshitz gravity [3]. Acknowledgement:

I would like to thank M. Haack for his very important remark that leaded to the correction of the first version of this paper. This work was supported by the Grant agency of the Czech republic under the grant P201/12/G028.

\section{References}

[1] O. Andreev, M. Haack and S. Hofmann, "On Nonrelativistic Diffeomorphism Invariance," arXiv:1309.7231 [hep-th].

[2] P. Horava, "Quantum Gravity at a Lifshitz Point," Phys. Rev. D 79 (2009) 084008 arXiv:0901.3775 [hep-th]].

[3] P. Horava, "General Covariance in Gravity at a Lifshitz Point," Class. Quant. Grav. 28 (2011) 114012 [arXiv:1101.1081 [hep-th]].

[4] S. Weinfurtner, T. P. Sotiriou and M. Visser, "Projectable Horava-Lifshitz gravity in a nutshell," J. Phys. Conf. Ser. 222 (2010) 012054 arXiv:1002.0308 [grqc]].

[5] T. P. Sotiriou, "Horava-Lifshitz gravity: a status report," J. Phys. Conf. Ser. 283 (2011) 012034 arXiv:1010.3218 [hep-th]].

[6] E. Gourgoulhon, "3+1 formalism and bases of numerical relativity," gr-qc/0703035 [GR-QC].

[7] R. L. Arnowitt, S. Deser, C. W. Misner, "The Dynamics of general relativity," gr-qc/0405109].

[8] P. Horava and C. M. Melby-Thompson, "General Covariance in Quantum Gravity at a Lifshitz Point," Phys. Rev. D 82 (2010) 064027 arXiv:1007.2410 [hep-th]].

[9] J. Kluson, "Lagrange Multiplier Modified Horava-Lifshitz Gravity," Eur. Phys. J. C 71 (2011) 1820 arXiv:1101.5880 [hep-th]].

[10] J. Kluson, "Hamiltonian Analysis of Non-Relativistic Covariant RFDiff Horava-Lifshitz Gravity," Phys. Rev. D 83 (2011) 044049 arXiv:1011.1857 [hep-th]]. 